\title{
Application of resonance Raman microscopy to in vivo carotenoid*
}

\author{
Chiasa Uragami ${ }^{1}$ Eiji Yamashita ${ }^{2}$, Andrew Gall ${ }^{3}$, Bruno Robert ${ }^{3}$ and Hideki Hashimoto ${ }^{1,4} \bowtie$ \\ 'Department of Physics and CREST/JST, Graduate School of Science, Osaka City University, Sugimoto, Sumiyoshi-ku, Osaka, Japan; ${ }^{2}$ Fuji Chemi- \\ cal Industry Co. Ltd., Nakaniikawa-gun, Toyama, Japan; 3Institute of Biology and Technology of Saclay, CEA-Saclay, Gif/Yvette, France, ${ }^{4 T h e}$ OCU \\ Advanced Research Institute for Natural Science and Technology (OCARINA), Osaka City University, Sugimoto, Sumiyoshi-ku, Osaka, Japan
}

The high antioxidant activity of astaxanthin has been attracted considerable attention in these days. One of the major antioxidant activities of this carotenoid is anti-photoaging. We have been focusing our attention on this particular issue. The anti-photoaging activity should be functioning in inner skin. In this study we tried to find out the fact that astaxanthin that has been swabbed on the outer surface of the skin has really passed through and reached to the inner skin. For this purpose resonance Raman microscopy was applied to the rat skin sample on which astaxanthin was swabbed on its outer surface. Astaxanthin gives rise to a unique Raman spectrum that is characteristic of its molecular structure. Therefore, we can easily identify the presence or absence of astaxanthin in the area of the rat skin that is subjected to this spectroscopic measurement. We used $532 \mathrm{~nm}$ laser light for probing the resonance Raman scattering of astaxanthin. Astaxanthin shows three strong Raman lines at 1508, 1145, and $993 \mathrm{~cm}^{-1}$. These three lines are ascribable to the $\mathrm{C}=\mathrm{C}$ stretching, $\mathrm{C}-\mathrm{C}$ stretching, and $\mathrm{C}-\mathrm{CH}_{3}$ in-plane rocking vibrational modes, respectively. We have constructed confocal Raman microscope that has the spatial resolution of ca. $500 \mathrm{~nm}$. Three-dimensional mapping of the Raman spectrum of astaxanthin has been performed in order to determine its distribution in the rat skin.

Key words: Astaxanthin, resonance Raman microscopy, in vivo carotenoid, Raman mapping

Received: 14 October, 2011; accepted: 01 March, 2012; available on-line: 17 March, 2012

\section{INTRODUCTION}

Astaxanthin widely distributes in marine organisms, for example, crustaceans (shrimp, crab) or some kinds of fish like salmon or sea beam and shows beautiful redorange color. It is already known that astaxanthin has many effects for human health, such as anti-inflammation, enhancing sport performance and endurance, limiting exercised induced muscle damage, attenuating eye fatigue, improving metabolic syndrome, anti-aging and anti-photoaging (Kurihara et al., 2002; Nijssen et al., 2002; Watanabe et al., 2007; Landrum, 2010). The specific effect that we focused our particular attention in this study is the anti-photoaging in skin.

The photoaging of skin would happen when UVA is irradiated to the inner skin. In this case reactive oxygen species (ROS) would be generated, and the ROS activates to produce singlet oxygen $\left({ }^{1} \mathrm{O}_{2}\right)$. The ${ }^{1} \mathrm{O}_{2}$ can readily destruct collagen, which keeps the skin tone and firmness, so the wrinkle may arrive with its presence. How astaxanthin works as the scavenger of the ${ }^{1} \mathrm{O}_{2}$ is due to the following way (Ichihashi et al., 2009; Hussein et al., 2006). Astaxanthin can directly react with ${ }^{1} \mathrm{O}_{2}$ as soon as it is generated, and as a result the triplet excited $\left(\mathrm{T}_{1}\right)$ state of astaxanthin is produced. The $\mathrm{T}_{1}$ species of astaxanthin thermally relaxes to the ground state without causing any harmful damages to the skins. Therefore, astaxanthin can prevent the destructive damage of collagen by the presence of ${ }^{1} \mathrm{O}_{2}$. This is a provisional mechanism of astaxanthin for anti-photoaging reagent, and could work at the inner skins. Therefore, it is primarily important to know the three dimensional distribution of astaxanthin in the inner skin before making a good quest to determine the detailed functional mechanisms of astaxanthin in the skins. In order to achieve this objective we have constructed confocal Raman microscope and applied it to observe the in vivo astaxanthin (Caspers et al., 2001; Darvin et al., 2006).

\section{MATERIALS AND METHODS}

The skin slices of rat were subjected to the Raman microscopy study. These slices were prepared after shaving the hair on back of the rat and swabbing AstaReal $50 \mathrm{~F}$ (Fuji chemical industry, Japan), the oil which contains $5 \%$ astaxanthin $(\mathrm{v} / \mathrm{v})$. We call the 0 hour $(0 \mathrm{~h})$ skin slice for the sample that was prepared immediately after the AstaReal $50 \mathrm{~F}$ was swabbed. The $3,6,12$ or 24 hour ( $3 \mathrm{~h}, 6 \mathrm{~h}, 12 \mathrm{~h}$ or $24 \mathrm{~h}$ ) skin slice is the sample that was prepared 3, 6, 12 or 24 hours after the AstaReal 50F was swabbed. The thickness of the skin slices slightly depends on the sample preparation but it is in the range between 8 and $10 \mu \mathrm{m}$. All of these preparations were performed at Kogure Laboratory, Kyoto Pharmaceutical University.

The Raman microscopy measurement was done using the home-built set-up whose block diagram is shown in Fig. 1. Figure 2 shows the absorption spectrum of alltrans astaxanthin in benzene solution at room temperature. Astaxanthin shows strong absorption band in the spectral region between $400-550 \mathrm{~nm}$. In order to fulfill the resonance condition for the Raman measurement we used a diode-pumped solid state CW-laser with output

e-mail: hassy@sci.osaka-cu.ac.jp

*Presented at the 16th International Symposium on Carotenoids, 17-22 July, 2011, Kraków, Poland

Abbreviations: CCD, charge-coupled device; CW, continuous wave; $\mathrm{ROS}$, reactive oxygen species; ${ }^{1} \mathrm{O}_{2}$, singlet oxygen. 


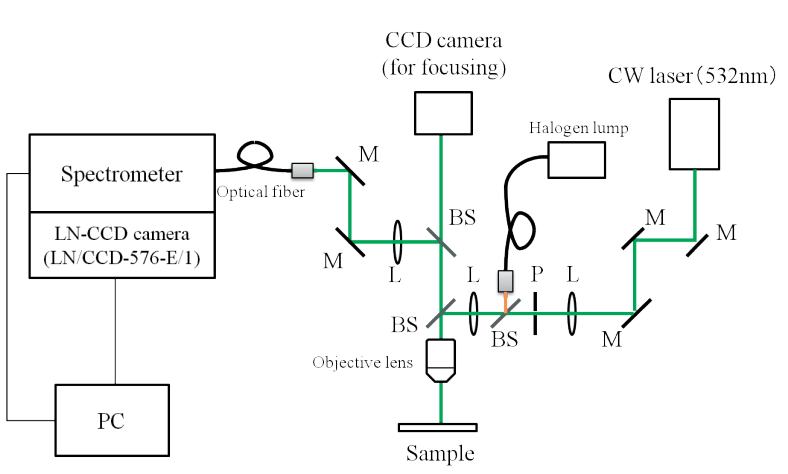

Figure 1. The block diagram of the experimental setup for confocal Raman microscopy.

Symbols are $M$, mirror; $L$, lens; $P$, pin-hole; $B S$, beam splitter.

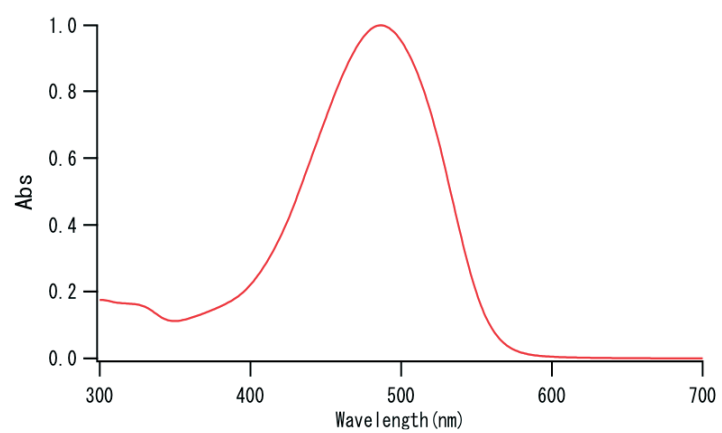

Figure 2. The absorption spectrum of astaxanthin in benzene.

at $532 \mathrm{~nm}$ (SDL-532-SLM-030T, Shanghai Dream Lasers Technology, China) for the excitation of Raman scattering. For the construction of three-dimension mapping images the electric translation stages along $\mathrm{X}, \mathrm{Y}$ directions (SGSP26-100(XY), Sigma Koki Corporation, Japan) and Z direction (SGSP80-20ZF, Sigma Koki Corporation, Japan) were used. These stages were computer controlled using a stage controller (SHOT-204MS, Sigma Koki Corporation, Japan). The Raman scattering was detected by using liquid nitrogen cooled CCD detector (LN/CCD-576-E/1, Roper Scientific, USA) after passing through a spectrometer (SpectraPro 2300i, Acton Research Corporation, USA) coupled to an optical fiber. The back-scattering optical geometry was adopted and all the measurements were performed at room temperature.

As illustrated in Fig. 1, confocal microscope configuration was achieved by the insertion of a pinhole aperture (25 $\mu \mathrm{m}$ diameter) between the excitation laser and the objective lens (SLWD Plan (APO), Atago Bussan, Japan) as well as the detection of Raman scattering through a single mode optical fiber (20 $\mu \mathrm{m}$ diameter). The present system guarantees the highest spatial resolution of $250 \mathrm{~nm}$ when using $\times 90$ objective lens (NA is $0.75)$. But typically the spatial resolution was $467 \mathrm{~nm}$ using $\times 40$ objective lens (NA is 0.42 ). Another CCD camera on top of the objective lens can detect the real time image of the samples under the Köhler illumination.

\section{RESULTS AND DISCUSSION}

Figure 3 shows the typical example of the Raman spectrum of astaxanthin in the rat skin. Because of the resonance condition Raman lines of astaxanthin are greatly enhanced, and hence the presence or absence of astaxanthin can readily be identified. Astax-

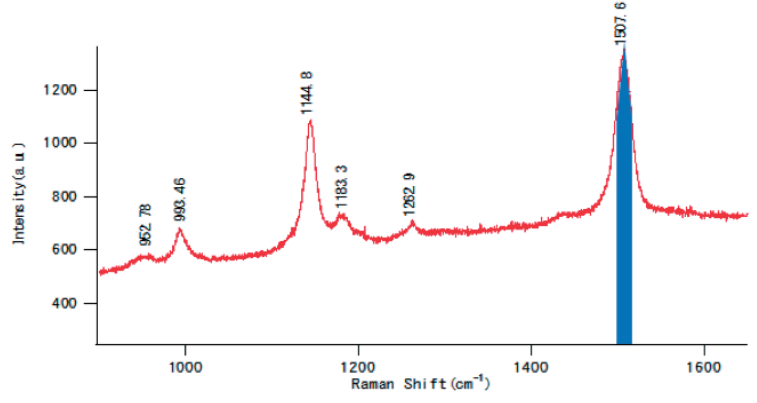

Figure 3. The Raman spectrum of astaxanthin in the rat skin. The blue part is the integrated part for mapping image.

anthin gives rise to three intense Raman lines at 1508 , 1145, and $993 \mathrm{~cm}^{-1}$. These Raman lines are assigned to the $\mathrm{C}=\mathrm{C}$ stretching, $\mathrm{C}-\mathrm{C}$ stretching and $\mathrm{C}-\mathrm{CH}_{3}$ inplane bending vibrational modes, respectively (Saito \& Tasumi 1983). The frequency of the $\mathrm{C}=\mathrm{C}$ stretching Raman line is sensitive to the molecular structure of carotenoids (Saito \& Tasumi, 1983, Merlin, 1985). Therefore this particular Raman line can be used to

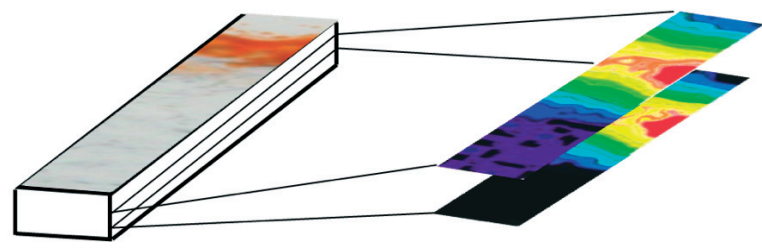

Figure 4. The scheme how to construct three-dimensional optical sectioning images of Raman scattering (Raman mapping) of astaxanthin in the rat skin.

The spatial resolution is typically $467 \mathrm{~nm}$ (highest resolution is 250 $\mathrm{nm}$ ) in the horizontal $(X-Y)$ directions and $3 \mu \mathrm{m}$ in the vertical (Z) direction.
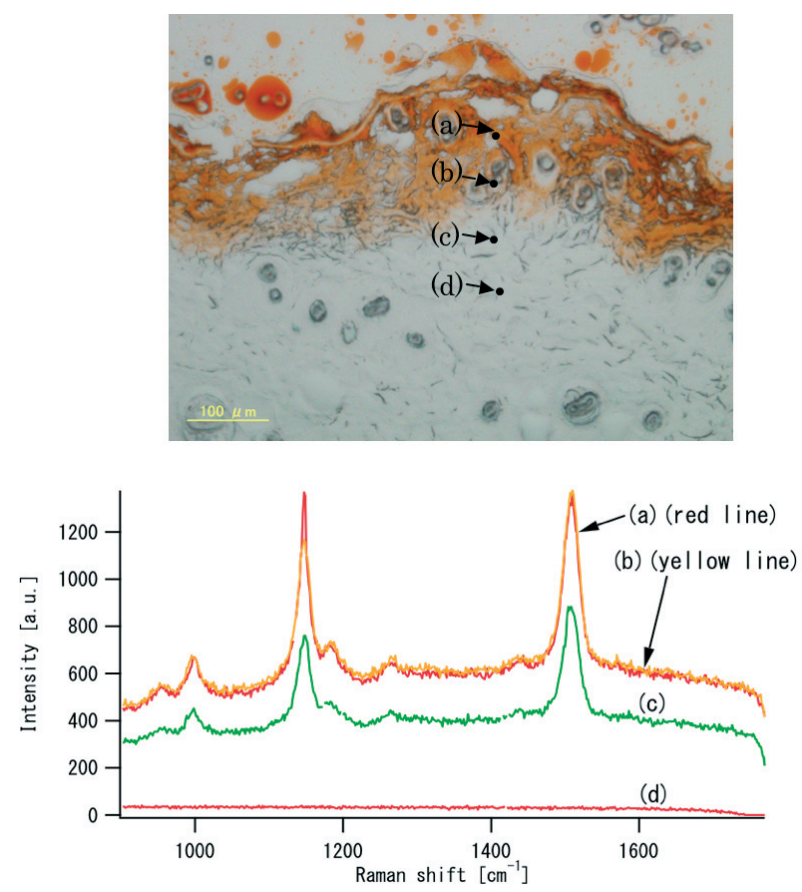

Figure 5. The photograph of $0 \mathrm{~h}$ rat skin slice (upper panel) and the Raman spectra observed at the marked positions

(a), (b), (c), and (d) with black dots in the photograph (lower panel). 

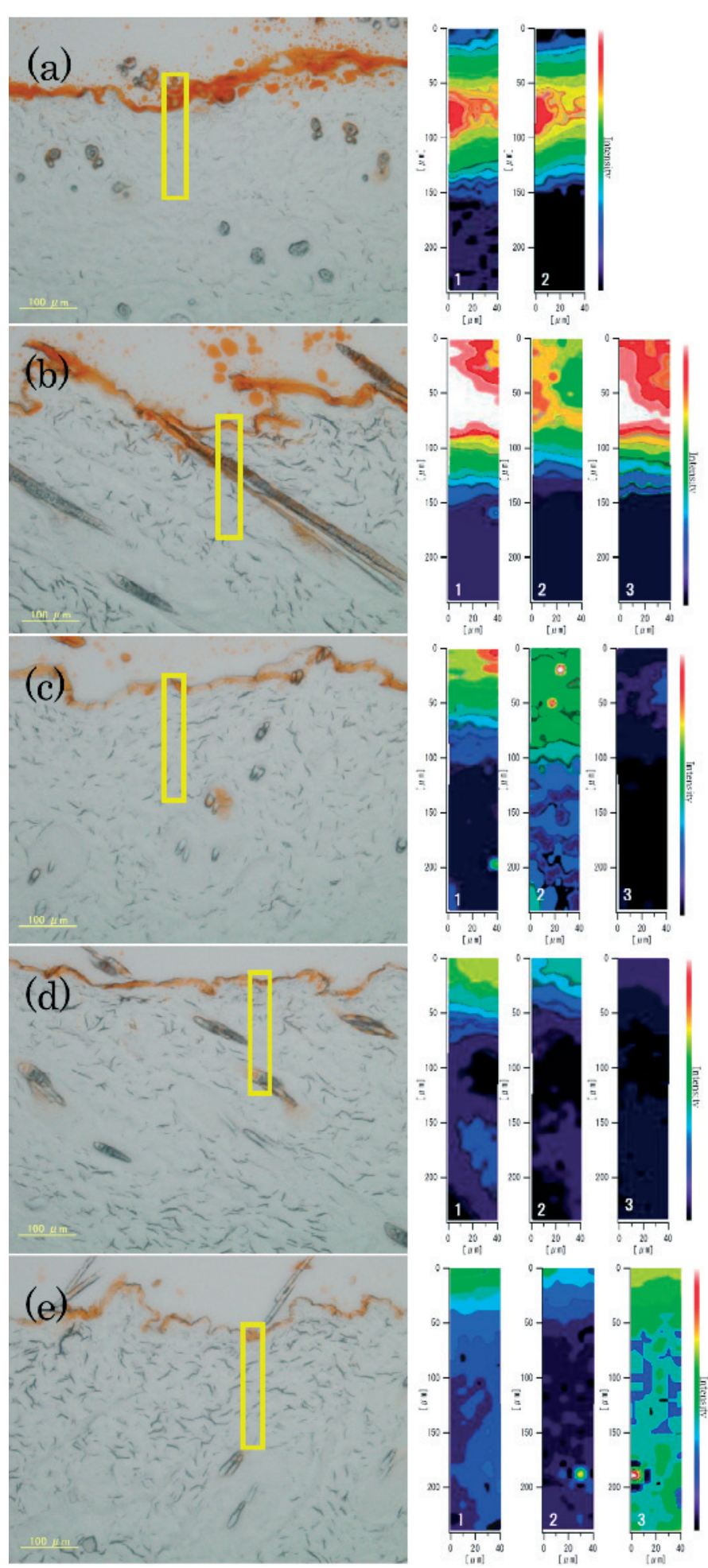

Figure 6. The photographs (left panels) and Raman microscopy images (right panels) of (a) $0 \mathrm{~h}$, (b) $3 \mathrm{~h}$, (c) $6 \mathrm{~h}$, (d) $12 \mathrm{~h}$, and (e) $24 \mathrm{~h}$ rat skin slices. The Raman microscopy images in the region of yellow squares in the photographs (left panels) are shown in the right panels. The Raman microscopy images are constructed using the integrated intensity of the $C=C$ stretching Raman line of astaxanthin. The Raman intensity is displayed with a color code of 10 degree of gradation. A warm colour shows the higher intensity of the Raman scattering and a cold colour shows the lower intensity. 1 and 2 in (a) show the optical slice images detected at the depth of 3 and $7 \mu \mathrm{m}, 1,2$ and 3 in (b), (c), and (d) show the images detected at the depth of 1, 3, and $7 \mu \mathrm{m}$, respectively, and 1,2, and 3 in (e) show the images detected at the depth of $0,4.5$ and $9 \mu \mathrm{m}$, respectively. The spatial resolution is $467 \mathrm{~nm}$ in the horizontal direction and $3 \mu \mathrm{m}$ in the vertical (depth) direction. In the horizontal (X-Y) direction Raman spectra were recorded point-by-point by translating the $X$ or $Y$ stages $8 \mu \mathrm{m}$ after each measurement. identify astaxanthin in the skin slice samples. In order to construct Raman mapping images the intensity of the $\mathrm{C}=\mathrm{C}$ stretching Raman line was integrated in the region of $\mathrm{cm}^{-1}$ of the peak frequency (see blue part in Fig. 3). Since the confocal microscope detects the light emitted from a small-irradiated volume, the skin slice samples were translated point-by-point and then the mapping images could be constructed. This is a well-recognized technique called as 'optical sectioning' in the research field of confocal microscopy (Dieing et al. 2010). This pointby-point translation was performed along $\mathrm{X}-\mathrm{Y}$ directions at each set depth direction (Z-direction, see Fig. 4).

Figure 5 shows the photograph of 0 h rat skin slice and Raman spectra detected at the positions of (a), (b), (c) and (d) in the photograph. The orange color part in the photograph corresponds to the outer skin region. The Raman spectra observed at the positions of (a) and (b) show equivalently the highest intensity signals from astaxanthin. This can be well accounted for since we can see the presence of astaxanthin by its orange color even with our naked eyes (see the photograph in Fig. 5). The Raman spectrum observed at the position (c) shows apparent intensity of the Raman signals from astaxanthin, although its color cannot be seen in the photograph. This is a good example to show the usefulness of Raman spectroscopy to probe the presence of astaxanthin in the rat skin samples. The position (c) is more than $100 \mu \mathrm{m}$ depth from the outer skin and it is obvious that we are probing the region of the inner skin. Therefore, this result is a strong indication that astaxanthin has already reached to inner skin just after swabbing on the outer skin surface. It is interesting to note that at the position of (d) (much deeper side in the inner skin) there is no clear indication of the presence of astaxanthin.

In order to make a good quest to determine the spatial distribution of astaxanthin in the rat skin we have constructed threedimensional mapping images of the Raman scattering from astaxanthin. Figure 6 shows the results of Raman mapping images together with the photographs of rat skin slices. In the Raman mapping images of 0 $\mathrm{h}$ skin slice, we could see that astaxanthin has already penetrated to more than $100 \mu \mathrm{m}$ from the outer surface of the rat skin (vide supra). This distribution of astaxanthin can also be seen in the preparation of $3 \mathrm{~h}$ skin slice (see Fig. 6(b)), but it gradually decreases along the time elapse (see Fig. 6(c) and (d)). In the sample of $24 \mathrm{~h}$ skin slice we could only see the presence of astaxanthin at the outer surface of the skin where the orange colour can be seen in the photograph of Fig. 6(e). The decrease of the distribution of astaxanthin can be ascribed to be due to the absorption or metabolism of astaxanthin in the inner skin. Further study is now in pro- 
gress in order to obtain the final answer to interpret this curious phenomenon.

\section{CONCLUSION}

Confocal Raman microscopy was applied to detect the three-dimensional distribution of astaxanthin in the rat skin. The present study clarifies that astaxanthin that was swabbed onto the outer surface of skin can very quickly penetrate into the inner skin. Resonance Raman microscopy is indeed a powerful tool to investigate the distribution of carotenoid molecules in the biological tissues.

\section{Acknowledgements}

We are grateful to Prof. Kentaro Kogure at Kyoto Pharmaceutical University for the preparation of Rat skin slices. HH thanks for HFSP and Nissan Science Foundation for financial support.

\section{REFERENCES}

Caspers PJ, Lucassen GW, Carter EA, Bruining HA, Puppels GJ (2001) In vivo confocal raman microspectroscopy of the skin: noninvasive determination of molecular concentration profiles. J. Invest. Dermat. 116: 434-442.
Darvin ME, Gersonde I, Albrecht H, Sterry W, Landemann J (2006) Non-Invasive in vivo detection of the carotenoid antioxidant substance lycopene in the human skin using the resonance Raman spectroscopy. Laser Phys Lett 3: 460-463.

Dieing T, Hollricher O, Toporski J (2010) Confocal Raman Microscopy, Pronger Series in Optical Sciences 158, Springer-Verlag, Berlin, Heidelberg, Germany.

Hussein G, Sankawa U, Goto H, Matsumoto K, Watanabe H (2006) Astaxanthin, a crotenoid with potential human health and nutrition. J Nat Prod 69: 442-449.

Ichihashi M, Ando H, Yoshida M, Niki Y, Matsui M (2009) Photoaging of the skin. Anti-Aging Med 6: 46-59.

Kurihara H, Koda H, Asami S, Kiso Y, Tanaka T (2002) Contribution of the antioxidative property of astaxanthin to its protective effect on the promotion of cancer metastasis in mice treated with restraint stress. Life Sci 20: 2509-2520.

Landrum JT (2010) Carotenoids: Physical, Chemical and Biological Functions and Properties, CRS Press, Florida, USA.

Merlin JC (1985) Resonance Raman spectroscopy of carotenoids and carotenoid-containing systems. Pure Appl Chem 57: 785-792.

Nijssen A, Bakker-Schut TC, Heule F, Caspres PJ, Hayes DP, Neumann MHA, Puppels GJ (2002) Discriminating basal cell carcinoma from its surrounding tissue by Raman spectroscopy. I Invest Dermat 119: $64-69$.

Saito S, Tasumi M (1983) Normal-coordinate analysis of $\beta$-carotene isomers and assignments of the Raman and infrared bands. I Raman Spectroscopy 14: 310-321.

Watanabe H, Hussein G, Goto H, Nakagawa T, Oda S, Matsumoto K, Sankawa U (2007) Astaxanthin improves metabolic syndrome in the rat model. Carotenoid Sci 11: 84-89. 